\title{
Absence of PRSS1 mutations and association of SPINK 1 trypsin inhibitor mutations in hereditary and non-hereditary chronic pancreatitis
}

\author{
G R Chandak, M M Idris, D N Reddy, K R Mani, S Bhaskar, G V Rao, L Singh
}

Gut 2004;53:723-728. doi: 10.1136/gut.2003.026526

See end of article for authors' affiliations

.....................

Correspondence to: G R Chandak, Genome Research Group, Centre for Cellular and Molecular Biology, Uppal Rd Hyderabad 500007 India; chandakgrc@ ccmb.res.in/chandakgrc@ yahoo.com

Accepted for publication 5 November 2003

\begin{abstract}
Background and aims: Mutations in the cationic trypsinogen (protease, serine, 1 (trypsin 1); PRSS1) gene are causally associated with recurrent acute and chronic pancreatitis. We investigated whether mutations in the PRSS1 gene are associated with hereditary and non-hereditary pancreatitis. As a modifier role has been proposed for trypsin inhibitor (serine protease inhibitor, Kazal type I; SPINK1) mutations, the role of SPINK1 mutations in these patients was also analysed.

Subjects and methods: The coding regions of PRSS1 and SPINK1 genes were sequenced in 290 controls and 198 patients, of whom 120 were diagnosed as idiopathic (ICP), 41 as alcoholic (ACP), and 37 as hereditary pancreatitis (HP). Twenty four unaffected relatives of HP probands were also analysed and genotype-phenotype correlations and statistical analyses were performed.

Results: No mutations in the PRSS1 gene were detected in any of the patients, including HP patients, while the N34S mutation was observed in the SPINK1 gene in the majority of HP patients $(73 \%)$. Similarly, $26.8 \%$ of ACP (11 of 41 ) and $32.5 \%$ (39 of 120) of ICP patients also had SPINK1 mutations. The N34S mutation was observed in both homozygous and heterozygous conditions. In comparison, only $2.76 \%$ of the control population had the N34S allele $(p<0.001)$. The P55S mutation was observed in one ICP and one ACP patient, and in three normal individuals. Genotype-phenotype correlations did not suggest any significant difference in the age of onset, severity of disease, or pancreatic endocrine insufficiency in patients with or without mutated SPINK1 and irrespective of the allelic status of N34S SPINK1.

Conclusions: Irrespective of the aetiology, mutations in the PRSS1 gene are not associated with chronic pancreatitis, including HP. In contrast, the N34S mutation in the SPINKI gene shows a significant correlation in these patients. A comparable phenotype in terms of age of onset, diabetes mellitus, and other phenotypic features in patients with or without SPINK1 mutations and N34S homozygotes and heterozygotes suggests that there may still be involvement of other genetic or environmental factors.
\end{abstract} hronic pancreatitis is a progressive inflammatory disease of the pancreas characterised by chronic inflammation and progressive fibrosis with loss of both exocrine and/or endocrine function. ${ }^{12}$ Alcohol is generally considered an important risk factor for the development of chronic pancreatitis. However, additional factors such as heredity, smoking, anatomical variations, and metabolic disorders (for example, hyperlipidaemia and hypercalcaemia) have also been identified. Close to one third of patients are classified as having idiopathic chronic pancreatitis (ICP) as no association with any of the aforementioned aetiological factors can be established. ${ }^{2}$ Irrespective of the aetiology, the clinical pattern of chronic pancreatitis is characterised by an early stage with recurrent episodes of acute pancreatitis (AP) followed by a late stage with pancreatic calcifications, pancreatic insufficiency, and diabetes mellitus in the majority of patients. ${ }^{3}$ The pathogenesis of chronic pancreatitis is unclear and it is unknown whether there are factors common to the aetiologically different forms of chronic pancreatitis. ${ }^{34}$

Chronic pancreatitis is generally considered as an autodigestive disease secondary to premature activation of trypsinogen within the pancreas although many studies have recently reported both isolated as well as syndromic autoimmune pancreatitis. ${ }^{5}$ The frequent association of this type of pyncreatis with autoimmune diseases such as sjogren syndrome suggests that an autoimmune mechanism may be involved in some patients with pancreatitis. ${ }^{3}$ Several genetic risk factors for chronic pancreatitis have been identified

Abbreviations: $A P$, acute pancreatitis; $A C P$, alcoholic chronic pancreatitis; CFTR, cystic fibrosis transmembrane regulator; HP, hereditary pancreatitis; ICP, idiopathic chronic pancreatitis; PRSS1, protease, serine, 1 (trypsin 1); PSTI/SPINK1, pancreatic secretory trypsin inhibitor/serine protease inhibitor, Kazal type I; TCP, tropical

recently. Two missense mutations, $\mathrm{R} 122 \mathrm{H}^{6}$ and $\mathrm{N} 29 \mathrm{I},{ }^{7}$ in the human cationic trypsinogen gene (protease, serine, l (trypsin 1); PRSS1) have frequently been detected in patients with hereditary pancreatitis (HP). The clinical significance and inheritance of several other rare PRSS1 variants such as Al6V, ${ }^{8}$ D22G, ${ }^{9}$ K23R, ${ }^{10}$ R122C, ${ }^{11}$ and $\mathrm{N} 29 \mathrm{~T}^{12}$ are poorly understood. Subsequent efforts to investigate the presence of HP associated PRSSI mutations have shown a very low incidence in $\mathrm{ICP}^{78^{13-15}}$ and complete absence in alcohol related pancreatitis (ACP). ${ }^{16}$ Recently, we showed absence of PRSS1 mutations in patients with tropical calcific pancreatitis (TCP), ${ }^{17}$ a type of idiopathic pancreatitis in the tropics, with a phenotype different than alcoholic pancreatitis. ${ }^{18}$

The underlying causes of variability in penetrance are not clear but observations indicate the involvement of environmental as well as other genetic factors. More recently, the N34S mutation of the serine protease inhibitor, Kazal type I (SPINK1) has been reported to be strongly associated with idiopathic and familial pancreatitis. ${ }^{13}{ }^{19}$ Subsequent studies however have reported a very low prevalence of the mutated calcific pancreatitis 
SPINKl gene in ICP patients, ${ }^{20-22}$ while in a recent study SPINKl mutations were observed in $47 \%$ of TCP patients. ${ }^{17}$ Although alcohol is a known predisposing factor for pancreatitis, only $5-10 \%$ of alcoholics develop the disease $\mathrm{e}^{23}$ who might have a genetic predisposition to develop pancreatitis. Witt and colleagues ${ }^{24}$ reported a $5.8 \%$ prevalence of N34S SPINKl in ACP patients, thus implicating mutated SPINKl in its pathogenesis. SPINKl acts as the first line of defence against prematurely activated trypsinogen by inhibiting approximately $20 \%$ of total trypsin activity within the pancreas. ${ }^{25}$ The role of SPINKl mutations, particularly the N34S mutation, is a matter of controversy, with some suggesting a causal ${ }^{13}$ while others advocating a modifier role for this molecule. ${ }^{19}$

The genetic basis of pancreatitis in India has rarely been explored and a few studies have focused only on TCP. ${ }^{17}{ }^{26} \mathrm{We}$ undertook a study on a large cohort of patients with hereditary and non-hereditary pancreatitis (ICP and ACP) to determine if PRSSI and SPINKI mutations are associated with chronic pancreatitis in India and also to understand their respective roles in the causation of the disease. We found no mutations in the PRSSl gene but detected only SPINKl mutations in HP as well as in ICP and ACP patients. We therefore propose a genetic basis of chronic pancreatitis (irrespective of its aetiology) in India, different from that observed in Western countries. The observations made in this study may have implications in counselling and modification of the predisposition risk by avoiding exposure to possible precipitating factors such as alcohol, smoking, nutritional, etc, in India.

\section{METHODOLOGY Selection of patients}

Patients were identified and investigated at the Asian Institute of Gastroenterology, Hyderabad. The diagnosis of chronic pancreatitis was based on at least two separate episodes of abdominal pain and radiological findings of pancreatic calcifications by computed tomography, endoscopic ultrasonography, and/or pathological findings such as pancreatic ductal irregularities and dilations on endoscopic retrograde cholangiopancreatography. ${ }^{3}$ A detailed questionnaire, including clinical and family history and various investigations, was collected from all patients and their unaffected relatives willing to participate in the study. Clinical history included aetiology, type and severity of pain, frequency of attacks, presence or absence of diabetes mellitus, and age at onset of symptoms and diabetes mellitus, etc. Exclusion criteria for a diagnosis of ICP included absence of precipitating factors such as alcohol, gall stones, infection, trauma, medications, and metabolic disorders, age over 65 years, and a positive family history. ${ }^{3}$ Diabetes mellitus was diagnosed at a fasting plasma glucose value of $>126 \mathrm{mg} /$ $\mathrm{dl}$ and a two hour plasma glucose value of $>200 \mathrm{mg} / \mathrm{dl}$, and/ or requirements for insulin or oral hypoglycaemic drugs. ${ }^{27}$ Alcohol was considered causal in chronic pancreatitis patients with a daily intake equivalent to more than $80 \mathrm{~g}$ of ethanol for at least two years. ${ }^{4} \mathrm{~A}$ diagnosis of HP was made on the basis of at least two affected first degree relatives or three or more second degree relatives in two or more generations. ${ }^{28}$

Thus, in total, 198 patients ( 120 ICP, 41 ACP, and $37 \mathrm{HP}$ ) and 24 unaffected relatives from HP families participated in the study. A total of 290 healthy volunteers from the same institute constituted the control population. Blood samples were withdrawn using EDTA as the anticoagulant after obtaining written informed consent. The institutional ethics committee approved the study following the Indian Council of Medical Research guidelines for research on human subjects.

\section{DNA studies}

All blood samples were analysed at the Centre for Cellular and Molecular Biology, Hyderabad, where a unique identification number was assigned to each sample and data fed into indigenously developed software for patient details. Genomic DNA was isolated from leucocytes following standard protocols. As there are no reports on the genetics of hereditary or non-hereditary pancreatitis in the Indian population, we decided to sequence PRSSI and SPINKI genes to screen for the reported mutations as well as for any novel variants. The coding and flanking non-coding regions of both genes were amplified using published primer sequences, ${ }^{613}$ as per protocols published previously. ${ }^{17}$ Sequencing was done on both strands using the Big dye terminator cycle sequencing ready kit on an ABI 310 genetic analyser (Applied Biosystems, Perkin Elmer New Jersey, USA). A total of 580 control alleles were also sequenced to identify the PRSSI and SPINKI variants and their prevalence in the general population.

\section{Statistical analysis}

All values are presented as median (range, 95\% confidence interval $(\mathrm{CI}))$. The $\chi^{2}$ test was used to analyse differences in the prevalence of SPINKl and N34S mutations among ICP, ACP, and HP patients, as well as in controls. We categorised the study cohort based on the presence or absence of the N34S SPINKl mutation and its zygosity. Phenotypic variability in features such as age of onset and presence or absence of diabetes mellitus, etc, among these groups were analysed by applying the Mann-Whitney U test. SPSS for windows software (SPSS Inc, Chicago, Illinois, USA) was used for analyses. A p value of less than 0.05 was considered statistically significant.

\section{RESULTS}

\section{Patient details}

Our study cohort comprised 37 HP patients from 16 families and 161 patients with non-hereditary pancreatitis (120 ICP and 41 ACP patients). There were 156 males and 42 females but all ACP patients were exclusively male. The majority of HP families $(n=13)$ had two or more first degree relatives with pancreatitis while three or more affected second degree relatives were observed in three families in two or more generations. Twenty four unaffected relatives of HP patients comprising 14 males and 10 females were also included. The majority of patients presented with pain in the abdomen $(88 \%)$ while diabetes mellitus was the presenting symptom in the rest. Median age of onset for HP and ICP patients was comparable at 24.5 and 23.5 years, respectively, which was significantly lower than 36 years for ACP patients $(\mathrm{p}<0.001)$. However, HP patients reported a longer duration of disease compared with ICP and ACP (table 1).

\section{DNA analysis}

We sequenced the entire coding region of PRSSI and SPINKI genes in all the patients and controls. None of the patients or controls carried the common mutations or any novel variant in the coding region of the PRSSl gene. However, two commonly reported neutral polymorphisms 162Asp $($ GAC $>$ GAT) and 246Asn (AAC $>$ AAT) were observed in the majority of patients $(88 \%)$ as well as in controls $(90 \%$; $\mathrm{p}>0.05)$. In comparison, 77 patients $(38.9 \%)$ with chronic pancreatitis had at least one SPINKI mutation. The majority of patients $(n=75)$ carried the N34S allele, including 15 homozygotes and 60 heterozygotes (table 2). The N34S mutation was found to be in complete linkage disequilibrium with IVS-37T $>$ C. P55S was observed in the heterozygous state in only two patients $(1.01 \%)$. The previously reported neutral polymorphism $-253 \mathrm{~T}>\mathrm{C}$ was identified in the 
Table 1 Characteristics of the study population

\begin{tabular}{lllll}
\hline & HP & ICP & ACP & Total \\
\hline $\mathrm{n}$ & 37 & 120 & 41 & 198 \\
Sex $(\mathrm{M} / \mathrm{F})$ & $28 / 9$ & $87 / 33$ & $41 / 0$ & $156 / 42$ \\
Age at presentation $(\mathrm{y})$ & $39.5(31.4-46.6)$ & $27.5(26.7-30.9)$ & $40.0(37.1-41.9)$ & $30.0(29.6-33.8)$ \\
Age at onset $(\mathrm{y})$ & $24.5(18.1-34.5)$ & $23.5(22.8-27.3)$ & $36.0(32.9-37.9)$ & $27.0(26.0-29.6)$ \\
Duration of symptoms $(\mathrm{y})$ & $9.5(6.7-15.9)$ & $4.7(3.9-5.4)$ & $4.0(3.7-5.7)$ & $5.3(4.5-6.0)$ \\
\hline
\end{tabular}

Values are median (range $95 \%$ confidence interval).

$\mathrm{n}$, number of patients; HP, hereditary pancreatitis; ICP, idiopathic chronic pancreatitis; ACP, alcohol related pancreatitis.

heterozygous state in $3.5 \%$ of patients and in $27.9 \%$ of controls. Eight of 290 healthy controls also carried the N34S mutation $(2.76 \%$, allele frequency $=0.014)$ while P55S was observed in only three individuals $(1.03 \%$, allele frequency $=0.0017$ ). Both mutations were present in the heterozygous state and no other previously reported mutations such as $2 \mathrm{~T}>\mathrm{C}, 41 \mathrm{~T}>\mathrm{C}$, etc, were detected in these patients.

Thirty eight of 120 ICP patients $(31.7 \%)$ carried the N34S mutation $(\mathrm{p}<0.0001 \quad v$ controls $)$, of which seven were homozygous. However, no significant difference in N34S SPINKI mutation frequency was noted for early onset $(35.7 \%)$ and late onset forms $(22.2 \% ; p=0.3872)$ of ICP. One P55S heterozygote was observed in one each of ICP and ACP patients. Interestingly, we identified the N34S mutation in 10 of 41 ACP patients (24.4\%; $<<0.0001 v$ controls), which is significantly higher compared with previous reports suggesting frequencies of $5.8 \%,{ }^{24} 6.0 \%,{ }^{29}$ and $5.6 \% .^{30}$ All were N34S carriers except one P55S heterozygote and one N34S homozygote. To date, no N34S homozygote has been reported in this group of patients. This individual was a 31 year old patient with persistent pain since the age of 20 years and diabetes for two years with very low alcohol intake for past five years. Although the diagnosis of ACP is based on a history of excessive alcohol intake on a background of recurrent attacks of $\mathrm{AP}$, the amount of alcohol intake was reported to vary from $25 \mathrm{~g} /$ day to more than $80 \mathrm{~g}$ /day for five years. ${ }^{4}$ Of 16 families matching the criteria of HP, N34S SPINKl mutation was detected in $12(75 \%)$. Twenty seven of 37 HP patients $(73.0 \% ; \mathrm{p}<0.0001 v$ controls) carried the N34S mutation, including seven homozygotes. Interestingly, all N34S homozygotes in this group were diabetic, with age of onset between 5 and 12 years. Of 24 unaffected relatives, six $(25 \%)$ carried the N34S SPINKl mutation, including one homozygote. The only homozygote was a 23 year old individual without pancreatitis or diabetes mellitus, although his heterozygous parents had the disease.

\section{Genotype-phenotype correlation}

We categorised the study cohort according to aetiology and then compared SPINKI N34S positive and negative patients in each category as a function of various phenotypic markers (table 3). Age at onset of symptoms was lower in the group with the N34S SPINKl compared with those carrying the wild-type SPINKl in each category but did not reach statistical significance, except in HP patients $(p=0.045)$. Analysis of N34S carrier frequency after categorising our study cohort into groups by age showed interesting results. The $<20$ year group had a carrier frequency of $52.8 \%$ (28 of 53 ), which was significantly higher than that of $24.8 \%$ (36 of $145)$ in the 20-65 year old group $(p<0.016)$. Interestingly, the majority of homozygotes (14 of 15) had chronic pancreatitis before the age of 20 years. The only homozygote in the older group was a 54 year old patient with mild disease and diabetes for six years. An increased influence of environmental factors in the latter group may have contributed to this significant difference. ${ }^{31}$

Diabetes mellitus as a feature of pancreatic endocrine insufficiency was equally prevalent in both groups, as were other parameters of disease severity such as pain, pseudocysts, pancreatic ductal abnormalities, etc. (table 3). Almost half of the patients with chronic pancreatitis and diabetes had the N34S SPINKl mutation (42.9\%) compared with only $36.5 \%$ of patients without diabetes (table 4 ). Relatively more N34S homozygotes were observed in patients with diabetes $(50 \%)$ than the group without diabetes mellitus (10.5\%). This may be due to additional genetic or environmental factors, especially in the HP cohort as the prevalence of the N34S mutation was comparable in both groups. However, the association between N34S and diabetes mellitus did not reach statistical significance in all three categories of patients. We did not find any patient with pancreatic carcinoma although many patients had a long duration of chronic pancreatitis.

Data from HP families showed a variable genotypephenotype association in individual families. In one of the families, the proband was a 40 year old woman with pancreatitis at 21 years and diabetes at 23 years (fig 1). She was detected to be homozygous for N34S SPINK1, which was inherited from her obligate heterozygous healthy parents. Her father had been diabetic for the past 30 years but of her two obligate N34S heterozygote sons, the younger has had

Table 2 Distribution and status of the PRSS1 and SPINK1 mutations in affected individuals

\begin{tabular}{lllll}
\hline & HP & ICP & ACP & Total \\
\hline$n$ & 37 & 120 & 41 & 198 \\
PRSS1 mutation* & - & - & - & - \\
SPINK1 mutation & $27(73 \%)$ & $39(32.5 \%)$ & $11(26.8 \%)$ & $77(38.9 \%)$ \\
N34S & 27 & 38 & 10 & $75(37.9 \%)$ \\
$\quad$ Homozygote & 7 & 7 & 1 & 15 \\
$\quad$ Heterozygote & 20 & 31 & 9 & 60 \\
P55S & - & 1 & 1 & $2(1.01 \%)$ \\
$\quad$ Homozygote & - & - & - & - \\
Heterozygote & - & 1 & 1 & 2
\end{tabular}

$\mathrm{HP}$, hereditary pancreatitis; ICP, idiopathic chronic pancreatitis; $A C P$, alcohol related pancreatitis; $n$, number of patients.

*Neither the common nor any novel mutation was detected in the PRSSI gene. 
Table 3 Distribution of various clinical parameters among patients with chronic pancreatitis based on N34S SPINK1 mutation status

\begin{tabular}{|c|c|c|c|c|c|c|}
\hline & \multicolumn{2}{|l|}{$\mathrm{HP}(n=37)$} & \multicolumn{2}{|l|}{ ICP $(n=120)$} & \multicolumn{2}{|l|}{$A C P(n=41)$} \\
\hline & Mutated SPINK1 & Mutation negative & Mutated SPINK1 & Mutation negative & Mutated SPINK1 & Mutation negative \\
\hline $\mathrm{n}$ & 27 & 10 & 38 & 82 & 10 & 31 \\
\hline $\begin{array}{l}\text { Age at presentation }(\mathrm{y}) \\
\text { (median, range } 95 \% \mathrm{Cl} \text { ) }\end{array}$ & $35.0(31.5-36.1)$ & $39.5(36.5-44.3)$ & $24.0(22.2-28.8)$ & $28.5(27.8-32.9)$ & $31.0(28.8-38.2)$ & $41.0(38.3-43.3)$ \\
\hline $\begin{array}{l}\text { Age at onset }(y)^{*} \\
\text { (median, range } 95 \% \mathrm{Cl} \text { ) }\end{array}$ & $23.5(21.2-29.2)$ & $24.5(23.2-31.8)$ & $19.5(17.8-24.2)$ & $25.0(24.6-29.7)$ & $28.0(24.2-34.5)$ & $38.0(34.8-40.0)$ \\
\hline \multicolumn{7}{|l|}{ Clinical parameters } \\
\hline Intermittent & $18(74.1 \%)$ & $3(30 \%)$ & $18(47.4 \%)$ & $19(23.2 \%)$ & $4(40 \%)$ & $5(16.1 \%)$ \\
\hline Constant & $6(22.2 \%)$ & $6(60 \%)$ & $14(36.8 \%)$ & $54(65.8 \%)$ & $4(40 \%)$ & $24(77.4 \%)$ \\
\hline Diabetes mellitus $†$ & $11(40.7 \%)$ & $4(40 \%)$ & $4(10.5 \%)$ & $15(18.3 \%)$ & $3(30 \%)$ & $5(16.1 \%)$ \\
\hline Smoking & $4(14.8 \%)$ & $3(30 \%)$ & $6(15.8 \%)$ & $22(26.8 \%)$ & $2(20 \%)$ & $8(25.8 \%)$ \\
\hline Pseudocyst & $4(14.8 \%)$ & $2(20 \%)$ & $6(15.8 \%)$ & $20(24.4 \%)$ & $2(20 \%)$ & $8(25.8 \%)$ \\
\hline $\begin{array}{l}n, \text { number of patients; HP, } \\
{ }^{*} p=0.045 \text { for HP; } p=0.17 \\
t p=0.978 \text { for HP; } p=0.50 \\
\text { Percentages refer to the pro }\end{array}$ & $\begin{array}{l}\text { ereditary pancreatiti } \\
\text { for ICP; } p=0.091 \\
5 \text { for ICP; } p=0.741 \\
\text { oortion of patients } \mathrm{W}\end{array}$ & $\begin{array}{l}\text { s; ICP, idiopathic chr } \\
\text { or ACP. } \\
\text { or ACP. } \\
\text { thin each group. }\end{array}$ & & alcohol related pa & eatitis. & \\
\hline
\end{tabular}

both pancreatitis and diabetes for past five years while the elder one is healthy. Three of her brothers were positive for N34S and had diabetes without evidence of pancreatitis. In another family, the proband was an 18 year old N34S heterozygote, inherited from his heterozygous father who also had an early onset of the disease. However, his elder brother and paternal grandmother are healthy despite being heterozygous for N34S SPINK1, whereas two aunts with N34S/WT have severe pancreatitis with diabetes and another heterozygote aunt has diabetes only.

\section{DISCUSSION}

Chronic pancreatitis is a heterogeneous disease and its genetic basis in India has not been investigated. We analysed 198 patients with hereditary and non-hereditary (idiopathic and alcoholic) pancreatitis with the major objective of understanding the respective roles of PRSSI and SPINKI mutations in its causation. As there is considerable confusion about the role of SPINKl mutations, we also looked at the association of these mutations and their zygosity with phenotype. In the present study, except for two previously reported cSNPs, ${ }^{7}$ no PRSSI mutation was identified in any patient or control individual. Absence of PRSS1 mutations in HP and ICP patients is intriguing as such mutations have been reported in up to $60 \%$ of $\mathrm{HP}^{32}$ and approximately $20 \%$ of ICP patients. ${ }^{33}$ We describe here for the first time the absence of PRSS1 mutations in Indian patients with chronic pancreatitis of different aetiologies, which confirm observations made previously in Indian TCP patients. ${ }^{17}{ }^{26}$ This is most likely related to their genetic makeup as no other study from abroad has reported on the absence of PRSSI mutations in $\mathrm{HP}$ as well as in non-hereditary chronic pancreatitis patients and a simultaneous strong association with SPINKl mutations. However, interaction with other factors, such as environmental and nutritional influences, may also play an important role. Although previous studies showing the presence of identical mutations in White HP kindreds as well as in Mongolians indicate heterogeneity but no racial specificity, ${ }^{34}$ our results suggest that such a phenomenon exists. Our findings strongly suggest that irrespective of its aetiology, established mutations in PRSSI are not a common cause of chronic pancreatitis in the Indian population.

However, SPINKl mutations were found to be strongly associated with all types of chronic pancreatitis. Most patients with a SPINKl variation had the N34S mutation and the prevalence was significantly higher in HP compared with ICP and ACP $(p<0.001)$. The presence of the N34S SPINKl mutation in the majority of HP patients is particularly interesting as PRSS1 mutations are lacking in these patients. N34S SPINKl prevalence $(2.76 \%)$ in controls was comparable with other studies from India ${ }^{26}{ }^{35}$ and higher than $1.5 \%$ found in the French population, ${ }^{20} 1.58 \%$ from $\mathrm{USA}^{19}$ and $0.36 \%$ from Germany ${ }^{13}$ but much lower than $4 \%$ in a healthy control population from Liverpool. ${ }^{29}$ Therefore, distribution of the N34S allele among various populations might be more variable than originally assumed. ${ }^{29}{ }^{36}$ The observed prevalence of mutated SPINKl in ICP patients $(32.5 \%)$ was significantly higher than the $6.4 \%,{ }^{37} 18.0 \%,{ }^{29}$ and $21.0 \%{ }^{30}$ in other studies but much less than $40.4 \%$ reported by Pfutzer and colleagues. ${ }^{19}$ Although a strong association has been reported between mutated SPINKl and familial ICP, but not with true ICP, ${ }^{29}$ we did not attempt to categorise this further as our diagnosis of ICP was considered in the absence of known aetiological factors, including heredity. A stronger genetic basis has recently been suggested for early onset rather than late onset $\mathrm{ICP}^{38}$ but no significant association for N34S SPINK1 prevalence was identified in this study, although the majority of patients in the older group (12 of 36) presented with diabetes compared with only seven of 84 early onset patients $(p=0.0103)$. The higher age of onset for

Table 4 Distribution of the N34S SPINK1 mutation in patients with chronic pancreatitis with respect to diabetes mellitus

\begin{tabular}{|c|c|c|c|c|c|c|c|c|}
\hline & \multicolumn{2}{|l|}{ HP $(n=37)$} & \multicolumn{2}{|c|}{ ICP $(n=120)$} & \multicolumn{2}{|c|}{$A C P(n=41)$} & \multicolumn{2}{|c|}{ Total $(n=198)$} \\
\hline & With DM & Without DM & With DM & Without DM & With DM & Without DM & With DM & Without DM \\
\hline$n$ & 15 & 22 & 19 & 101 & 8 & 33 & 42 & 156 \\
\hline N34S SPINK1 mutation & $11(73.3 \%)$ & $16(72.7 \%)$ & $4(21 \%)$ & $34(33.7 \%)$ & $3(37.5 \%)$ & $7(21.2 \%)$ & $18(42.9 \%)$ & $57(36.5 \%)$ \\
\hline Homozygote & 7 & - & 1 & 6 & 1 & - & 9 & 6 \\
\hline Heterozygote & 4 & 16 & 3 & 28 & 2 & 7 & 9 & 51 \\
\hline
\end{tabular}

$\mathrm{n}$, number of patients; HP, hereditary pancreatitis; ICP, idiopathic chronic pancreatitis; ACP, alcohol related chronic pancreatitis; DM, diabetes mellitus. Values in parentheses indicate percentages. 

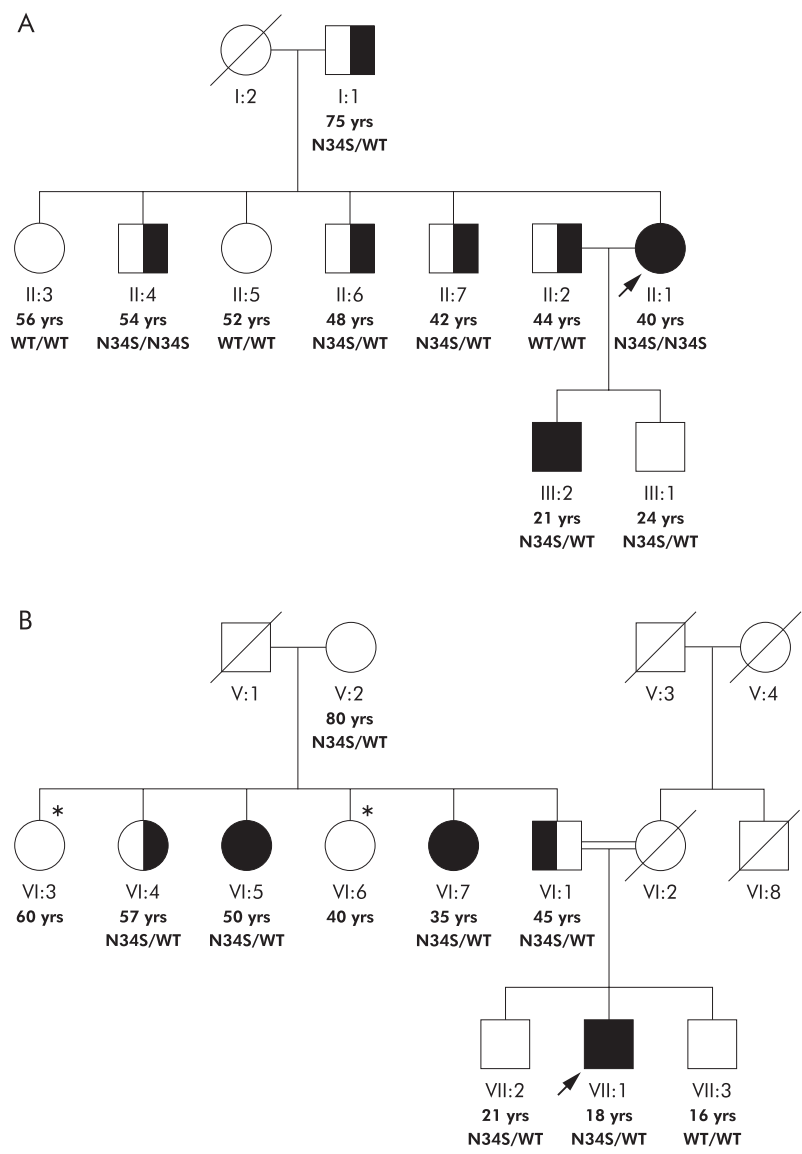

Diabetes mellitus

Both chronic pancreatitis and diabetes mellitus $₫$ Proband

* DNA samples not available

Figure 1 Two pedigrees of families with hereditary pancreatitis (HP) and coinheritance of the N34S mutation in the SPINK1 gene. N34S/ N34S, homozygotes; N34S/WT, heterozygotes; WT/WT, wild-type.

HP patients (24.5 years) in our study compared with the majority of studies conducted abroad may be due to the presence of N34S SPINK1 mutations which are hypothesised to perform a modifier role in comparison with the causal role played by PRSS1 mutations in Western HP patients. ${ }^{19} \mathrm{~A}$ highly significant prevalence of SPINKl mutations in our cohort of ACP patients suggests an important role for this genetic variant in our population. Decreased trypsin inhibitor to trypsinogen levels have been reported in the pancreatic juice of alcoholics compared with controls without alcoholism. ${ }^{39}$ Alcohol may also affect SPINKl regulation during the complex inflammatory processes in human alcoholic pancreatitis. ${ }^{40}$ Earlier studies have shown that in comparison with White patients, Black patients are $2-3$ times more likely to be hospitalised for chronic pancreatitis than alcoholic cirrhosis. ${ }^{41}$ Thus, alcoholics in India may be more susceptible to chronic pancreatitis due to a combination of factors such as genetic makeup, racial difference in diet, type or quantity of alcohol, or smoking, etc. Although the present knowledge suggests that ACP patients are likely to have higher interactions with environmental factors in comparison with other types of chronic pancreatitis, there is a strong genetic basis for ACP patients in India. As SPINKI mutations appear to predispose humans to an earlier age of onset, ${ }^{19}$ they may have an impact on the phenotypic presentation of ACP.

The cohort in our study represents the conglomeration of chronic pancreatitis patients usually seen in routine clinical practice in developing countries such as India. It has been a matter of intense speculation how a mutated inhibitor, especially N34S SPINKl, can cause the disease in the presence of wild-type PRSS1 where the R122 autolysis site is intact. PRSS1 mutations such as $\mathrm{R} 122 \mathrm{H}$ and N29I have been shown to lead to either failure of autolytic degradation of active trypsin or facilitated activation of trypsinogen. This pancreatic protease-protease inhibitor system may play an important role in the pathogenesis of chronic pancreatitis as SPINKl can block $\sim 20 \%$ of trypsin activity. ${ }^{25}$ Although the exact mechanism is not well understood, it is hypothesised that mutated inhibitor, with N34S mutation, may have functional consequences, probably due to alteration of the protein structure. $^{19}$

Current research on the role of N34S SPINK1 is confusing, with both a causal as well as a modifier role being described. ${ }^{201319}$ A recent study supports the significance of SPINKl mutations based on disappearance of the pancreas in homozygous knockout mice of SPINKl, although heterozygous mice showed no alteration in pancreatic tissue (as quoted in Hirota and colleagues ${ }^{42}$ ). We did not observe any significant difference in the phenotype between SPINKl mutation positive and mutation negative groups, or between SPINK1 N34S heterozygotes and homozygotes, also reported in Indian TCP patients by us. ${ }^{17}$ Although the association between N34S and diabetes mellitus was not statistically significant in all three categories of patients, N34S homozygosity was positively associated with diabetes mellitus. This suggests that the N34S SPINKI mutation may be involved in only modifying the phenotype.

Several studies have argued about SPINKl mutations being autosomal recessive or autosomal dominant. Our results may suggest an autosomal dominant mode of inheritance with a low level of penetrance. At the same time, the autosomal recessive model is also suggested by the high prevalence of N34S homozygotes $(7.6 \%)$ in patients. Despite a significantly strong association between N34S and HP, analysis of HP families in our study showed variable inheritance patterns and associations with the phenotype. This phenomenon of genetic heterogeneity is characteristic of complex diseases with an important role for the environment. Hence, it may be logical to suggest that the N34S mutation significantly lowers the threshold for pancreatitis due to other genetic or non-genetic factors, including the environment. Based on the finding that the vast majority of these patients are N34S SPINKl heterozygotes, other genes such as cystic fibrosis transmembrane regulator (CFTR) ${ }^{43-45}$ and $\alpha_{1}$-antitrypsin have been speculated to act as modifier genes. ${ }^{46}{ }^{47}$ It may be interesting to screen these patients for CFTR mutations. Although the frequency of CFTR gene mutations is low in our population, it may be tempting to speculate that the coexistence of mild mutations in CFTR with the N34S mutation could account for the low penetrance of SPINK1. The association between ICP and SPINK1, PRSS1, and CFTR genes in recent studies ${ }^{48} 49$ suggests a genetic basis and challenges the concept of ICP as a non-genetic disorder. This confusion in understanding of hereditary chronic pancreatitis needs to be addressed appropriately in future studies so that a uniform terminology can be coined for these patients.

In conclusion, the N34S mutation in the SPINKl gene is strongly associated with chronic pancreatitis, although the penetrance is very low. However, mutations in the cationic trypsinogen gene are not an important cause of pancreatitis in the Indian population. To date, the N34S SPINKI 
mutation is the only factor implicating a genetic basis for chronic pancreatitis in Indian patients. This may have implications in presymptomatic genetic testing but analysis of more such patients are needed to validate such a conclusion.

\section{ACKNOWLEDGEMENTS}

The authors express sincere thanks to all the patients, their families, and individuals who voluntarily participated in the study. The study was supported by grant-in-aid from the Indian Council of Medical Research, India. Thanks are also due to Drs Yogendra Sharma and Ramesh K Aggarwal for their valuable suggestions in the manuscript.

\section{Authors' affiliations}

G R Chandak, M M Idris, K R Mani, S Bhaskar, L Singh, Genome Research Group, Centre for Cellular and Molecular Biology, Hyderabad, India

D N Reddy, G V Rao, Asian Institute of Gastroenterology, Medinova Diagnostic Services, Punjagutta, Hyderabad, India

\section{REFERENCES}

1 Ammann RW. Natural history of chronic pancreatitis. Dig Surg $1994 ; 11: 267-74$

2 Steer ML, Waxman I, Freedman S. Chronic pancreatitis. N Engl J Med 1995; 332:1482-90.

3 Etemad B, Whitcomb DC. Chronic pancreatitis:diagnosis, classification, and new genetic developments. Gastroenterology 2001;120:682-707.

4 Ammann RW. A clinically based classification system for alcoholic chronic pancreatitis: summary of an international workshop on chronic pancreatitis. Pancreas 1997:14:215-21.

5 Kloppel G, Luttges J, Lohr M, et al. Autoimmune pancreatitis: Pathological, clinical and immunological features. Pancreas 2003;27:14-19.

6 Whitcomb DC, Gorry MC, Preston RA, et al. Hereditary pancreatitis is caused by a mutation in the cationic trypsinogen gene. Nat Genet 1996;14:141-5.

7 Gorry MC, Gabbaizedeh D, Furey W, et al. Mutations in the cationic trypsinogen gene are associated with recurrent acute and chronic pancreatitis. Gastroenterology 1997; 113:1063-8.

8 Witt $\mathrm{H}$, Luck W, Becker M. A signal peptide cleavage site mutation in the cationic trypsinogen gene is strongly associated with chronic pancreatitis. Gastroenterology 1999;117:7-10.

9 Teich N, Ockenga J, Hoffmeister A, et al. Chronic pancreatitis associated with an activation peptide mutation that facilitates trypsin activation. Gastroenterology 2000;1 19:461-5.

10 Ferec $\mathrm{C}$, Raguenes $\mathrm{O}$, Salomon $\mathrm{R}$, et al. Mutations in the cationic trypsinogen gene and evidence for genetic heterogeneity in hereditary pancreatitis. J Med Genet 1999;36:228-32

11 Le Marechal C, Chen JM, Quere I, et al. Discrimination of three mutational events that result in a disruption of the R122 primary autolysis site of the human cationic trypsinogen (PRSS1) by denaturing high performance liquid chromatography. BMC Genet 2001;2:19

12 Pfutzer R, Myers E, Applebaum-Shapiro S, et al. Novel cationic trypsinogen (PRSS1) N29T and R122C mutations cause autosomal dominant hereditary pancreatitis. Gut 2002:50:271-2.

13 Witt H, Luck W, Hennies HC, et al. Mutations in the gene encoding the serine protease inhibitor, Kazal type 1 are associated with chronic pancreatitis. Nat Genet 2000;25:213-16.

14 Pfutzer RH, Whitcomb DC. Trypsinogen mutations in chronic pancreatitis. Gastroenterology 1999;117:1507-8.

15 Chen JM, Raguenes O, Ferec C, et al. A CGC>CAT gene conversion-like event resulting in the $\mathrm{R} 122 \mathrm{H}$ mutation in the cationic trypsinogen gene and its implication in the genotyping of pancreatitis. J Med Genet 2000;37:E36.

16 Monaghan KG, Jackson CE, KuKuruga DL, et al. Mutation analysis of the cystic fibrosis and cationic trypsinogen genes in patients with alcohol-related pancreatitis. Am J Med Genet 2000;94:120-4.

17 Chandak GR, Idris MM, Reddy DN, et al. Mutations in the pancreatic secretory trypsin inhibitor gene (PSTI/SPINK1) rather than the cationic trypsinogen gene (PRSS1) are significantly associated with tropical calcific pancreatitis. J Med Genet 2002;39:347-51.

18 Mohan V, Chari ST, Hitman GA, et al. Familial aggregation in tropical fibrocalculous pancreatic diabetes. Pancreas 1989;4:690-3

19 Pfutzer RH, Barmada MM, Brunskill APJ, et al. SPINK1/PSTI polymorphisms act as disease modifiers in familial and idiopathic chronic pancreatitis. Gastroenterology 2000;119:615-23.
20 Chen JM, Mercier B, Audrezet MP, et al. Mutational analysis of the human pancreatic secretory trypsin inhibitor (PSTI) gene in hereditary and sporadic chronic pancreatitis. J Med Genet 2000;37:67-9.

21 Kaneko K, Nagasaki Y, Furukuwa T, et al. Analysis of the human pancreatic secretory trypsin inhibitor (PSTI) gene mutations in Japanese patients with chronic pancreatitis. J Hum Genet 2001;46:293-7.

22 Ockenga J, Dork T, Stuhrmann M. Low prevalence of SPINK1 gene mutations in adult patients with chronic idiopathic pancreatitis. J Med Genet 2001;38:243-4.

23 Bisceglie AM, Segal I. Cirrhosis and chronic pancreatitis in alcoholics. J Clin Gasteroenterol 1984;6:199-200.

24 Witt H, Luck W, Becker M, et al. Mutation in the SPINK1 trypsin inhibitor gene, alcohol use, and chronic pancreatitis. JAMA 2001;285:2716-17.

25 Horii A, Kobayashi T, Tomita N, et al. Primary structure of human pancreatic secretory trypsin inhibitor (PSTI) gene. Biochem Biophys Res Commun 1987; 149:635-41.

26 Bhatia E, Choudhuri G, Sikora SS, et al. Tropical calcific pancreatitis:strong association with SPINK1 trypsin inhibitor mutations. Gastroenterology 2002;123:1020-5.

27 The Expert Committee on the Diagnosis and Classification of Diabetes Mellitus. Diabetes Care 1997;20:1 183-97.

28 Perrault J. Hereditary pancreatitis. Gastroenterol Clin North Am 1994;23:743-52.

29 Threadgold J, Greenhalf W, Ellis I, et al. The N34S mutation of SPINK1 (PSTI) is associated with a familial pattern of idiopathic chronic pancreatitis but does not cause the disease. Gut 2002:50:675-81.

30 Drenth JP, te Morsche R, Jansen JB. Mutations in serine protease inhibitor Kazal type 1 are strongly associated with chronic pancreatitis. Gut 2002; 50:687-92

31 Pfutzer RH, Whitcomb DC. SPINK 1 mutations are associated with multiple phenotypes. Pancreatology 2001;1:457-60.

32 Dasouki MJ, Cogan J, Summar ML, et al. Heterogeneity in hereditary pancreatitis. Am J Med Genet 1998;77:47-53.

33 Creighton J, Lyall R, Wilson DI, et al. Mutations of the cationic trypsinogen gene in patients with chronic pancreatitis. Lancet 1999:354:42-3.

34 Nishimori I, Kamakura K, Fujikawa-Adachi K, et al. Mutations in exon 2 and 3 of the cationic trypsinogen gene in Japanese families with hereditary pancreatitis. Gut 1999:44:259-63.

35 Hassan Z, Mohan V, Ali L, et al. SPINK1 is a susceptibility gene for fibrocalculous pancreatic diabetes in subjects from the Indian subcontinent. Am J Hum Genet 2002;71:964-8.

36 Witt H. Gene mutations in children with chronic pancreatitis. Pancreatology $2001 ; 1: 432-8$

37 Chen JM, Mercier B, Audrezet MP, et al. Mutations of the pancreatic secretory trypsin inhibitor (PSTI) gene in idiopathic chronic pancreatitis. Gastroenterology 2001;120:1061-2.

38 Truninger $\mathrm{K}$, Witt $\mathrm{H}$, Kock, et al. Mutations of the serine protease inhibitor, Kazal type 1 gene, in patients with idiopathic chronic pancreatitis. Am J Gastroenterol 2002;97:1133-7.

39 Rinderknecht $\mathbf{H}$, Stace NH, Renner IG. Effects of chronic alcohol abuse on exocrine pancreatic secretion in man. Dig Dis Sci 1985:30:65-71.

40 Gukovskaya AS, Mouria M, Gukovsky I, et al. Ethanol metabolism and transcripton factor activation in pancreatic acinar cells in rats. Gastroenterology 2002;122:106-18

41 Lowenfels AB, Maisonneuve P, Grover $\mathrm{H}$, et al. Racial factors and the risk of chronic pancreatitis. Am J Gastroenterology 1999:94:790-4.

42 Hirota $M$, Kuwata K, Ohmuraya M, et al. From acute to chronic pancreatitis: The role of mutations in the pancreatic secretory trypsin inhibitor gene. J Pancreas 2003;4:83-8.

43 Sharer N, Schwarz M, Malone G, et al. Mutations of the cystic fibrosis gene in patients with chronic pancreatitis. N Engl J Med 1998;339:645-52.

44 Cohn JA, Friedman KJ, Noone PG, et al. Relation between mutations of the cystic fibrosis gene and idiopathic pancreatitis. N Engl J Med 1998:339:653-8.

45 Noone PG, Zhou Z, Silverman LM, et al. Cystic fibrosis gene mutations and pancreatitis risk: relation to epithelial ion transport and trypsin inhibitor gene mutations. Gastroenterology 2001;121:1310-9.

46 Witt $\mathrm{H}$, Kage A, Luck W, et al. Alphal-antitrypsin genotypes in patients with chronic pancreatitis. Scand J Gastroenterol 2002;37:356-9.

47 Teich N, Walther K, Bodeker H, et al. Relevance of variants in serum antiproteinases for the course of chronic pancreatitis. Scand J Gastroenterol 2002;37:360-5

48 Audrezet MP, Chen JM, Le Marechal C et al. Determination of the relative contribution of three genes - the cystic fibrosis transmembrane conductance regulator gene, the cationic trypsinogen gene, and the pancreatic secretory trypsin inhibitor gene-to the etiology of idiopathic chronic pancreatitis. Eur J Hum Genet 2002;10:100-6.

49 Perri F, Piepoli A, Andriulli A. On the role of CFTR, PRSS1 and PST1/SPINK1 in idiopathic chronic pancreatitis. Eur J Hum Genet 2003;11:107. 